\title{
Botulinum neurotoxin in cervical dystonia revisited - recent advances and unanswered questions
}

\author{
Jarosław Sławek ${ }^{1,2^{*}}$, Wolfgang H. Jost ${ }^{3^{*}}$ \\ ${ }^{1}$ Division of Neurological and Psychiatric Nursing, Faculty of Health Sciences, Medical University of Gdansk, Poland \\ ${ }^{2}$ Department of Neurology and Stroke, St. Adalbert Hospital, Gdansk, Poland \\ ${ }^{3}$ Parkinson-Klinik Ortenau, Wolfach, Germany
}

\begin{abstract}
Cervical dystonia (CD) usually presents a complex pattern of head/neck movements accompanied by tremor, myoclonic jerks and a wide spectrum of non-motor disturbances such as pain, depression, anxiety, and sleep problems. This is the most challenging indication for botulinum neurotoxin (BoNT) treatment. It can offer significant improvement, but it can be difficult after the first injection. Thorough examination and identification of the proper CD pattern, the identification of the muscles responsible, and adjusting doses given precisely under ultrasound and/or electromyographic guidance seem to be the key success modifiers. Nevertheless, this is a lifelong treatment and should be planned and conducted carefully to avoid failures and drop outs. The aim of this paper was to examine the current concepts in terms of anatomy, physiology and CD patterns (Col-Cap concept) as well as the proper dosages and any possible obstacles impeding successful treatment.
\end{abstract}

Key words: botulinum neurotoxin, cervical dystonia, electromyography, ultrasonography, deep brain stimulation

(Neurol Neurochir Pol 2021; 55 (2): 125-132)

\section{Introduction and aim}

Cervical dystonia $(\mathrm{CD})$ remains the most challenging indication for botulinum neurotoxin (BoNT) treatment. However effective, the improvement is usually suboptimal and unsatisfactory for many patients. In recent years, the approach to this treatment has evolved, with new concepts emerging regarding muscle involvement in the clinical patterns of CD (the Col-Cap concept) [1]. Unfortunately, for this new classification we do not have any proper scales to rate the improvement. Non-motor symptoms accompanying motor presentation may not respond to BoNT and can result in worse self-assessment of patients despite the head/neck correction. Guided injections (electromyography EMG, and ultrasound US), currently considered to be the standard approach, lack proof of their superiority over treatment based on anatomical landmarks and clinical judgment only. We still do not know the optimal dose per muscle. The growing number of toxins with different pharmacological properties and different dosing regimens (BoNT/A as onabotulinumtoxinA: ONA-BoNT/A, abobotulinumtoxinA: ABO-BoNT/A and incobotulinumtoxinA: INCO-BoNT/A and BoNT/B as rimabotulinumtoxinB: RIMA-BoNT/B) renders this problem even more complicated. Refractory cases should be assessed carefully for pseudoresistance, and deep brain stimulation (DBS) may be an alternative treatment [2].

However, the American Academy of Neurology Task Force has placed $\mathrm{ABO}-\mathrm{BoNT} / \mathrm{A}$ and RIMA-BoNT/B at level $\mathrm{A}$, and ONA- and INCO-BoNT/A at level B [3]. The recently published report of the Cochrane Library stated: "We are moderately certain in the evidence that a single BONT/A treatment session resulted in a clinically relevant reduction of $\mathrm{CD}$ specific impairment, and pain, and highly certain that it is well tolerated, compared with placebo. There are no data from RCTs evaluating the effectiveness and safety of repeated $\mathrm{BtA}$ injection cycles. There is no evidence from RCTs to allow us to draw definitive conclusions on the optimal treatment intervals and doses, the usefulness of guidance techniques

Address for correspondence: Jarosław Sławek, MD, PhD, Department of Neurology and Stroke, St. Adalbert Hospital, Al. Jana Pawła II 50 Str., $80-462$ Gdansk, Poland; e-mail: jaroslaw.slawek@gumed.edu.pl

${ }^{*}$ Both authors equally participated in the preparation of this manuscript. 
for injection, the impact on quality of life, or the duration of treatment effect." [4]

The aim of this paper was to revisit the treatment concepts of $\mathrm{CD}$ in terms of recently published papers, and look for unresolved questions to be addressed in future studies.

\section{History and clinical examination}

Over the last 30 years, BoNT has become established as the therapy of choice for CD. Early therapeutic work on CD considered it to be synonymous with spasmodic torticollis, and therapeutic recommendations corresponded mainly to the rotatory form. As a consequence, the sternocleidomastoid muscle and the contralateral splenius capitis muscle were injected. The good results of this early work led to approval studies being conducted, and then to approval being granted for the relevant muscles in rotatory spasmodic torticollis. Injection at that time was done according to clinical considerations, landmarks, and occasionally under EMG guidance [5].

In the last decade however, new considerations have become relevant: on the one hand more muscles are now being injected, and on the other hand a good many novel sub-forms have been identified. Another step forward concerns major progress in the use of US technology. One problematical aspect here is how to prove that with differentiating sub-forms and with the help of extensive diagnostic tools, results would be much better. Nonetheless, it is overall advantageous when recent developments are kept in mind for the purposes of medical training and, even more so, for improving on therapeutic attempts $[1,6,7]$.

Since the introduction of a new classification system and of new techniques, a number of very useful modifications and extensions have been established in recent years for taking the medical history as well as for the clinical examination $[1,6,7]$. The patient is interviewed as to their initial symptoms, the course of their development, ensuing symptoms (such as pain) and sensory tricks. Keeping the Col-Cap classification in mind, the primary relevant information on CD is obtained as soon as the patient enters the examination room and sits opposite the physician during anamnesis. Importantly, the patient should be seated parallel to the physician (not, as is too often the case, at an oblique angle) and should remove necklaces, scarves or turtle neck pullovers so that the position of the head and neck can be fully assessed. This usually suffices to evaluate the relevant head mispositioning and any possible sensory tricks.

At examination, a seat without a backrest is best used so that the patient does not have the opportunity to lean back, and also so that the physician can walk completely around him or her, or alternatively the patient can turn around while seated [8]. The patient should be asked to close his or her eyes so as to avoid possible positional correction through visual control and should be prevented from returning the head to the neutral position. In case of rotation of the trunk or raising one shoulder, a third person can best fixate that shoulder. The patient is then requested to demonstrate a sensory trick (geste antagoniste). The function and its possible reduction can be examined by having the patient rotate their head in the different planes of movement: flexing it ventrally and laterally, and extending it dorsally. Subsequently, the patient is examined while standing and while walking so as to judge movement and the influence of walking behaviour on the position of the head and possible compensatory mechanisms. This is followed by a clinical examination of the head position and the planes of movement, the muscle tonus and strength [8].

In cases of tremor-predominating types (e.g. 'no-no,') careful observation of tremor disappearance when turning the head to one side may help to establish the dominant direction of the dystonia. On the other hand, enhancement of tremor may suggest the activity of compensatory muscles.

After this initial examination, one must classify properly the subtype(s) of $\mathrm{CD}[1,6,7]$. It is very rare that movements are found at one level alone; a combination of several forms is more common. It is frequently quite difficult to judge which movements are the paramount ones, which are the resultant compensatory ones, and which muscles play an agonistic or antagonistic role in the different subtypes [7]. Therefore, our personal practice is to limit ourselves to the lead pattern and the relevant muscles. In subsequent treatments, doses and muscle selection will be adjusted. The muscles to be injected are selected depending on the type of CD under examination. In complex cases, electromyography (EMG) is also useful for better differentiation between active and non-active muscles. This is especially true for muscles without a phasic component, but EMG may be useful in identifying muscles involved in tremor, where compensatory contraction is less likely to be a confounder. The EMG examination is likewise best done under ultrasound guidance.

\section{Anatomy and classification}

While in previous decades we selected muscles for injection based on the form of $\mathrm{CD}$, today we pay more attention to anatomical considerations. While earlier we made use of a deductive (top-down) methodology, today we apply rather an inductive (bottom-up) or individual approach [6,7]. Muscle origins, their insertions, and their functions, are the basics required for understanding $\mathrm{CD}$ patterns. According to the so called 'Col-Cap concept', three dimensions of movement in two levels can be distinguished $[1,6]$. From a functional point of view, there are two levels in which we differentiate the upper level between the skull and C2 and the lower one between $\mathrm{C} 2$ and $\mathrm{C} 7$, with the $\mathrm{C} 2$ vertebra regarded as a kind of fixed point. Dystonic activity of muscles with C2 (and above) and skull insertions form the so-called 'caput' and those with insertions below $\mathrm{C} 2$ and vertebral (or other) insertions are responsible for the so-called 'collis' subtypes (Fig. 1) In addition to the classical definitions of laterocollis, retrocollis, 


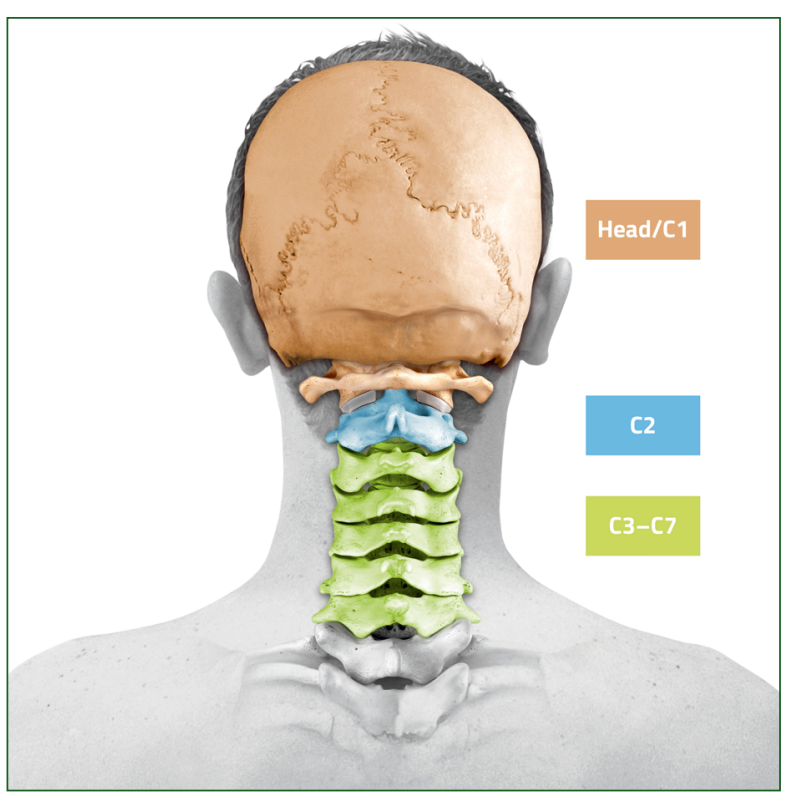

Figure 1. Anatomical basis of Col-Cap concept: Two levels of movement with $\mathrm{C} 2$ as a fixed point (modified as per Jost W. Atlas of Botulinum Toxin Injection, 3rd edition, KVM Verlag, Berlin, 2019)

anterocollis, and rotatory torticollis, we must add laterocaput, retrocaput, antecaput, and rotatory torticaput. Furthermore, three different forms of shift, as a combination of simple patterns, should be added. Lateral shift is a combination of laterocollis to one side and laterocaput to the opposite side; anterior shift is a combination of anterocollis and retrocaput; and retroshift is a combination of retrocollis and antecaput $[6,7]$. Data regarding this extended list of patterns can be found in our previous papers [5, 6].

It is not yet clear whether certain muscles constitute a single functional unit, or rather whether there are complete muscle chains. We do now know that some muscles are preferentially involved and that certain combinations present together more frequently $[9,10]$. In a recent study of 306 patients, splenius capitis was the most commonly involved muscle (83\%), followed by sternocleidomastoid (79.1\%) and trapezius (58.5\%). This was followed by levator scapulae, semispinalis capitis, and obliquus capitis inferior in $38.2 \%, 48.7 \%$ and $35.3 \%$ respectively. In torticaput, three muscles were injected in over half of the patients, splenius capitis ( $88 \%$ ), sternocleidomatoid $(84 \%)$, and trapezius $(60.7 \%)$ [9].

The most common primary form was torticaput (49\%), followed by laterocaput (16.7\%). Within this group, $16.3 \%$ of the patients had only one subtype of $\mathrm{CD}, 40.2 \%$ had two subtypes, $24.5 \%$ had threesubtypes, and $19 \%$ had four or more subtypes [10]. In the whole group, only $9.8 \%$ presented with laterocollis, and $8.8 \%$ with torticollis. All other subforms made up less than $5 \%$. The frequency of head tremor was $57.6 \%$, and torticaput was the most common dystonic subtype associated with tremor [11]. Many simple subtypes formed a complex pattern.
Torticaput $(\mathrm{n}=150)$ was combined in $46 \%$ with laterocaput, and in $20.7 \%$ with retrocaput. Furthermore it was combined in $18.7 \%$ with torticollis, and $12.7 \%$ with laterocollis. Laterocaput, the second most common primary pattern $(n=51)$, was combined mainly with torticaput (45.1\%), laterocollis (33.3\%), retrocaput $(23.5 \%)$, and antecollis (15.7\%). Laterocollis $(\mathrm{n}=30)$ was accompanied by laterocaput in $67.7 \%$, torticaput in $46.7 \%$, and in $16.7 \%$ by antecollis or retrocaput. The highest positive correlations were found for retrocaput with retrocollis [10].

The mean number of injected muscles in all patients was 4.2 (SD 1.6). In patients with torticaput, it was 4.4 (SD 1.6), in laterocaput 4.4 (SD 1.4), in laterocollis 4.0 (SD 1.4), and in torticollis $(\mathrm{n}=27) 4.2(\mathrm{SD} 1.8)$ [9].

\section{BoNT/A dose per muscle and per session}

In the majority of studies, the doses per muscle or the total dose per session have been established arbitrarily. The recommendations published to date are not based on relevant studies finding the proper dose, but on the clinical experience of experts and pre-established doses used in the pivotal clinical studies and then adopted in summary product characteristics (SPC). According to the SPCs, it is recommended that for ONA-BoNT/A, a maximal dose of $200 \mathrm{U}$ should be administered at first and should not exceed $300 \mathrm{U}$ in the following treatment sessions [12]. There is a similar recommendation for INCO-BoNT/A [13]; for ABO- BoNT/A, the recommended starting dose is $500 \mathrm{U}$ with a subsequent possibility to increase up to $1,000 \mathrm{U}$ if appropriate and if no dysphagia has been observed at the lower dose [14]. The US Food and Drug Administration recommends doses up to $400 \mathrm{U}$ for both ONA- and INCO-BoNT/A, 1,000 U for ABO-BoNT/A, and 10,000 U for RIMA-BoNT/B [15].

In a group of 305 patients representing real life treatment with stable and effective doses in the previous sessions, 154 patients received ONA-BoNT/A, 53 patients INCO-BoNT/A, and 98 patients ABO-BoNT/A. The mean total doses for a treatment session with ONA-, INCO- and ABO-BoNT/A were 159.5 U (SD = 62.4), 173.4 U (SD = 99.2), and $652.5 \mathrm{U}(\mathrm{SD}=285.5)$ respectively. The doses injected into each muscle in the ONA- or INCO-BoNT/A groups were between $19.7 \mathrm{U}$ and $49.2 \mathrm{U}$. The highest dose was injected into the splenius capitis, $49.2 \pm 26.0 \mathrm{U}$, with the highest total dose per session being $130 \mathrm{U}$. The doses in the ABO-BoNT/A group were between 75.4 and $139.6 \mathrm{U}$ per muscle, with the highest dose injected into the splenius capitis: $139.6 \pm 80.7 \mathrm{U}$ [16]. These real-life doses were lower than those recommended by experts $[17,18]$. We assume that the use of US guidance in our group [16] may be an advantage, and that more precise injections may result in lower doses being used subsequently, although this is only an indirect supposition. Keeping doses at effective, but also not too high, levels is recommended because doses that are too high may 
result in the formation of neutralising antibodies in longterm treatment [19].

\section{Injection and the use of EMG and US}

Since the very beginning of BoNT therapy, injections have been conducted taking into consideration anatomical landmarks. The use of EMG has become a well established tool. It improves the precision of the injection, especially the accuracy of needle placement within active muscles $[5,20]$. Thus, it may also improve safety and efficacy. It allows identification of the target and the most active muscles, but is unable to differentiate between 'dystonic' and 'non-dystonic' (compensatory) muscles.

At present US still competes with EMG [5]. Strictly speaking, there is no actual competition for the better position, but rather a debate on which gives better training for medical personnel. US outlines the anatomical structures more exactly [21], while EMG portrays function better. The two methods are thus not substitutes for one another, but rather complement each other (visibility and function) [5,22].

In addition, US, in improving the precision of injection, thereby improves safety and possibly efficacy. Furthermore, it allows for standardisation, because we can record in detail just which muscles we are injecting with which doses. During training, we can adequately portray the muscles involved, as well as their relationships to other muscles and structures such as nerves, vessels and bones. We have learned that muscles can often differ between individuals, and that individual muscles can be very thin (e.g. trapezius), meaning that a precise injection can only be guaranteed with the help of US. This is the only way to offer assurance that BoNT is applied precisely where we intend it [21-23].

We are more successful now in pinpointing injections, and in reaching muscles which we could not target earlier [22-25]. As a case in point, we should mention OCI (obliquus capitis inferior) which plays an essential role in the most frequent form of $C D$ and which used to be only rarely targeted for injection [22, 26].

In summary, we have found that US is indispensable and that EMG is useful in complicated cases and in combination with US [22].

Nevertheless, an unresolved problem remains regarding the clinical superiority of guided injections versus blinded ones, as we do not have comparative studies showing better results achieved by guided injections. However, in cadaver studies, the accuracy of needle placement was $100 \%$ with US vs. $79.2 \%$ without US for superficial muscles and $95.8 \%$ vs. $54.2 \%$ for deep muscles (with statistical significance) [25]. This should translate into a better clinical effect, but we need further studies performed on living patients.

One study assessed the impact of monitoring techniques such as US and/or CT in a small group of eight patients requiring injections in deep cervical muscles (obliquus capitis inferior, longus colli, obliquus capitis superior, scalenus anterior and scalenus posterior). The Tsui Scale confirmed a significant improvement occurring within four weeks (11.75 vs. 1.50) and on the TWSTRS (Toronto Western Spasmodic Torticollis Rating Scale) in each of the subscales (20.0 vs. 5.25, 20.0 vs. 7.0, and 13.1 vs. 6.5) [27].

\section{Non-motor symptoms and rating scales}

In addition to abnormal head postures, many patients present with so-called non-motor symptoms (NMS) [28-30]. It is a matter of debate whether these are direct or indirect symptoms of the disease. Non-motor symptoms have been reported in several studies: lack of self-confidence due to stigmatisation (61.8\%), sleep problems (59.8\%), and fatigue (51\%) [28]. In the study by Sławek et al., the authors reported depression in $47.5 \%$ of patients, and this was the major determinant of poor quality of life. Furthermore, after BoNT/A injection, the size effect for motor improvement in TWSTRS was favourable (1.1 SD 0.6) and much lower (0.5 SD 0.7) for depression (Montgomery-Asberg Depression Rating Scale) [31]. This might suggest that abnormal head posture and stigmatisation have no indirect impact and that depression is part of the spectrum of symptoms of $\mathrm{CD}$ itself. This was further proved by Berardelli et al. in 2015 when the authors demonstrated that after five years the successful treatment of motor problems (a statistically significant reduction in TWSTRS from 33.4 \pm 11.1 to $26.9 \pm 10.9$ ) did not correlate with an improvement of neuropsychiatric symptoms (65\% vs. 64\%) [32]. In a recent publication by Klingelhöfer et al., pain, insomnia and stigma were most prevalent, and emotional well-being and pain had a major impact on quality of life. Most NMS, with the exception of pain, stigma and daily activity, did not correlate with motor severity [29].

Self-awareness of motor dysfunction is higher in CD than in other patients with dyskinesias (e.g Parkinson's and Huntington's Diseases) and groups have been matched for depression, which may explain the poor impact of $\mathrm{CD}$ on emotional well-being [33].

Stamelou et al. concluded that NMS are not mere epiphenomena of dystonia and demand the same level of attention as motor ones. They should be considered in future pathophysiological models of dystonia [34].

Therefore, in conclusion we have to assess improvement based both on motor and NMS. Unfortunately, evaluation using the old version of TWSTRS and Tsui score did not consider NMS [35]. They are however taken into consideration in the new version of the TWSTRS, but this does not include the Col-Cap classification. Basically, the decision has to be made as to whether all symptoms should be considered, or only those symptoms which are improved by specific therapeutic measures. Any future rating scale must satisfy these requirements.

Lack of improvement of NMS despite good head position after BoNT injection may be responsible for treatment failures and drop outs in long-term treatment. 


\section{Treatment failures}

Supnet et al. noted long-term treatment discontinuation in $36.2 \%$ of a relatively large group of patients. Fifty seven percent of them described suboptimal effect, high costs and long distance to the treatment centre. Eight per cent reported the lack of any improvement, only $47 \%$ had regular treatment sessions every 12 weeks, and the results were statistically worse in males [36]. Treatment failures include a long list of possible causes from the misidentification of proper $C D$ pattern, muscle profiles, doses per muscle, side effects, and technical issues (too deep injections, too short needles used, no treatment guidance like EMG or US) to the formation of neutralising antibodies. Also, secondary or symptomatic dystonias as well as pseudodystonias have to be considered if the treatment is not effective. Pseudodystonias were revisited by Berlot et al. in 2019 and new disorders e.g. fibrodysplasia ossificans progressiva have been recognised as possible misdiagnoses $[5,37,38]$.

Fresh insights into possible treatment failures were noted by Hefter et al. who analysed how disease progression during treatment may influence the outcome. They included 74 patients with a mean time of treatment of 9.9 years. Mean improvement of $\mathrm{CD}$ reported by the patients and scored by the physician was about $50 \%$. The frequency of all symptoms (abnormal head position, reduced mobility, head tremor, muscle tension, pain) increased with duration of therapy. The longer the gap between the onset of symptoms and the onset of BoNT therapy, the poorer the long-term outcome, independent of the duration of BoNT treatment [39]. Another group noted in a cohort of 149 patients followed for 14 years the spread of dystonia from isolated to more complex forms in 23.5\% cases [36]. In a series of studies, Marciniec et al. reported that compared to moderate/good treatment satisfaction, CD patients with none/low BoNT efficacy had increased incidence of cervical pain, enhanced mean VAS score for pain, and higher coexistence of oromandibular dystonia (spread of dystonia). In addition, worse treatment satisfaction correlated with enhanced scores of Tsui, TWSTRS, as well as TWSTRS subscales: severity, disability and pain $[40,41]$. Neutralising antibodies may still be a real problem: Hefter et al. noted them in $16.2 \%$ of patients in long-term treatment. This was correlated with higher doses of BoNT used, longer treatment, and higher scores in CD severity scales [39].

We must systematically assess our patients at subsequent sessions, looking for new patterns evolving or new muscles involved, and adjust our injection regimens accordingly.

In a case of treatment failure at the onset of treatment, or if there is a loss of initial good effect, other treatment options such as DBS should be considered. If the patient did not respond from the very beginning, we recommend an algorithm and consultation at a secondary or tertiary movement disorder centre experienced in BoNT therapy (Fig. 2).

\section{Duration of symptom relief between injections}

It is a well-established rule to treat patients no more frequently than every 12 weeks. So-called booster injections (it was a common practice in the early years to inject patients with an additional dose after several weeks) have been identified as the chief culprit of antibody formation and secondary nonresponsiveness. Many patients have a shorter beneficial period (mean 10.5 weeks in $88 \%$ of patients) [42], and therefore physicians are tempted to inject earlier. They may be encouraged by no reports on antibody formulation after new BoNT/A preparation without complexing proteins (INCO-BoNT/A) [43]. Nevertheless, despite the potential risk of antibody formation, it may also result in dose accumulation and adverse effects such as dysphagia or muscle weakness. Walter et al., in a long-term treatment $[9.8$ \pm 6.2 years (range, $0.5-30$ years; adherence, $70.6 \%$ with 31.2 \pm 22.5 (3-112) treatment cycles], showed that independent risk factors for neutralising antibodies were high BoNT dose per treatment, switching between onabotulinumtoxinA and other BoNT formulations (except for switching to incobotulinumtoxinA), and treatment of neck muscles. They did not find antibodies in a group of 49 patients treated with incobotulinumtoxinA for up to 14 years. The authors recommended the use of the lowest possible dose for $\mathrm{CD}$ patients and avoiding unnecessary switching between formulations. The mean cumulative dose over time was a risk factor for antibodies formulation after ABO-, but not ONA- or INCO-BoNT/A [19]. This means also that we should avoid shortening the time between injections. On the other hand, ABO-BoNT/A injections for $\mathrm{CD}$ or spastic paresis $(500-1,000 \mathrm{U})$ had prolonged efficacy, and $72.6 \%, 77 \%$ and $81.5 \%$ of patients did not require injections at week 12 in three consecutive cycles. Moreover, $22.6 \%, 26.5 \%$ and $22.8 \%$ respectively did not require injections even after 24 weeks [44]. In real life, however, Supnet et al. reported that only $47 \%$ received injections every 12 weeks [36].

\section{Alternative treatments in refractory cases}

Pallidal DBS seems to be the most effective alternative treatment option for refractory patients $[45,46]$. The authors of a systematic review of 18 studies concluded that both surgical peripheral denervation and DBS are associated with a significant reduction in absolute TWSTRS total score, with no significant difference in the magnitude of reduction observed between the two treatments [47]. A small series of patients confirmed the long-term treatment effects (10-12.8 years): $C D$ scores improved by $53 \%$ (total TWSTRS); by $54.1 \%$ (severity score); and by $70.1 \%$ (disability score). Pain did not improve significantly. Improvement was stable over time. Patients with a tonic pattern of $C D$ responded less to $D B S$ than patients with a phasic pattern, and the effects were unrelated to aetiology [48]. According to analysis by the Cochrane group, severity of symptoms of CD after DBS GPi has been reduced across studies, but quality of life and safety concerns are still uncertain [2]. 


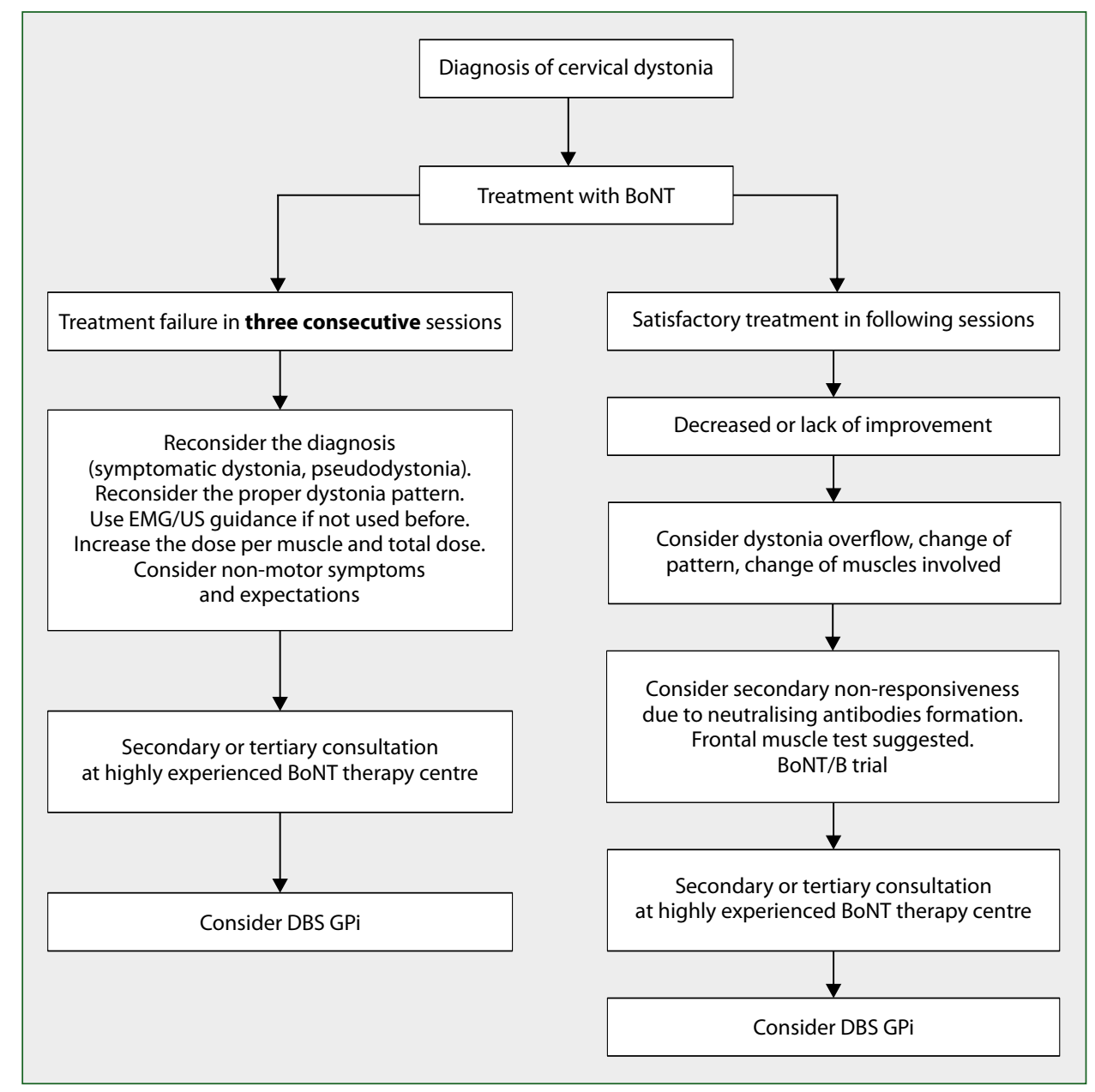

Figure 2. Algorithm for identification of possible treatment failures when starting and continuing treatment of patients with cervical dystonia

The unmet need seems to be a lack of clear protocols (as in Parkinson's Disease) regarding patient selection.

The next interventional procedure is selective muscle denervation. Wang et al. recently published a large series of 648 patients operated on successfully between 1995 and 2013, with a significant improvement observed between preoperative and postoperative TWSTRS evaluation $(73.5 \pm 11.9 \%)$ [49]. These results are in concordance with the earlier (between 1988 and 1996) study by the Mayo Clinic Group with a longterm follow up of a mean 3.4 years in 130 patients. The original level of moderate-to-excellent improvement in head position and pain was retained in at least 71 patients (70\%) [50]. Both these studies were retrospective. In the era of DBS, further prospective studies are required to confirm these results in randomised and blinded studies.

\section{Conclusions}

Botulinum neurotoxins offer effective treatment of $\mathrm{CD}$, but challenges remain. The Col-Cap concept seems convincing.
However, it is not widely used, nor are US/EMG guided injections. One possible obstacle seems to be the lack of well-documented studies showing Col-Cap superiority over the traditional approach. The lack of specific rating scales is probably one of the reasons.

Treatment protocols should also take into consideration non-motor symptoms (especially neuropsychiatric and sensory ones like pain) and the real expectations of patients. Also, unresolved issues include the proper dose per muscle/session and the correct treatment interval so as to avoid pseudo- or secondary failures (too small or too high doses, potentially resulting in lack of efficacy in the former or antibody formation in the latter). Alternative treatment options such as DBS GPi should be offered after careful selection of refractory patients, but there is as yet no consensus on selection and outcome protocols. Further studies are needed to answer all these unresolved questions.

Conflict of interest: Both authors are consultants for Allergan, Ipsen and Merz. 


\section{References}

1. Reichel G. Cervical dystonia: A new phenomenological classification for botulinum toxin therapy. Basal Ganglia. 2011; 1(1): 5-12, doi: 10.1016/j.baga.2011.01.001.

2. Rodrigues FB, Duarte GS, Prescott D, et al. Deep brain stimulation for dystonia. Cochrane Database Syst Rev. 2019; 1: CD012405, doi: 10.1002/14651858.CD012405.pub2, indexed in Pubmed: 30629283.

3. Simpson DM, Hallett M, Ashman EJ, et al. Practice guideline update summary: Botulinum neurotoxin for the treatment of blepharospasm, cervical dystonia, adult spasticity, and headache: Report of the Guideline Development Subcommittee of the American Academy of Neurology. Neurology. 2016; 86(19): 1818-1826, doi: 10.1212/ WNL.0000000000002560, indexed in Pubmed: 27164716.

4. Rodrigues FB, Duarte GS, Marques RE, et al. Botulinum toxin type A therapy for cervical dystonia. Cochrane Database Syst Rev. 2020; 11: CD003633, doi: 10.1002/14651858.CD003633.pub4, indexed in Pubmed: 33180963.

5. Tyślerowicz M, Kiedrzyńska W, Adamkiewicz B, et al. Cervical dystonia - improving the effectiveness of botulinum toxin therapy. Neurol Neurochir Pol. 2020; 54(3): 232-242, doi: 10.5603/PJNNS.a2020.0021, indexed in Pubmed: 32285434.

6. Jost WH, Tatu L. Selection of muscles for botulinum toxin injections in Cervical Dystonia. Mov Disord Clin Pract. 2015; 2(3): 224-226, doi: 10.1002/mdc3.12172, indexed in Pubmed: 30838229.

7. Tatu L, Jost WH. Anatomy and cervical dystonia : „Dysfunction follows form". J Neural Transm (Vienna). 2017; 124(2): 237-243, doi: 10.1007/s00702-016-1621-7, indexed in Pubmed: 27624726.

8. Jost W. Botulinumtoxintherapie der zervikalen Dystonie - Schritt für Schritt. Neurologie up2date. 2019; 2(02): 125-130, doi: 10.1055/a0821-0686.

9. Jost WH, Biering-Sørensen Bo, Drużdż A, et al. Preferred muscles in cervical dystonia. Neurol Neurochir Pol. 2020; 54(3): 277-279, doi: 10.5603/PJNNS.a2020.0022, indexed in Pubmed: 32227332.

10. Jost WH, Tatu L, Pandey S, et al. Frequency of different subtypes of cervical dystonia. J Neural Transm. 2020; 127: 45-50.

11. Pandey S, Kreisler A, Drużdż A, et al. Tremor in Idiopathic Cervical Dystonia - Possible Implications for Botulinum Toxin Treatment Considering the Col-Cap Classification. Tremor Other Hyperkinet Mov (N Y). 2020; 10: 13, doi: 10.5334/tohm.63, indexed in Pubmed: 32775027.

12. Allergan, Ltd. BOTOX® 100 U. Summary of product characteristics [webpage on the Internet]. Surrey, UK: Datapharm Communications Ltd; 2013 [updated December 12, 2012]. Available from: http://www. medicines.org.uk/emc/medicine/112. Accessed October. ; 8: 2020.

13. Dysport $\circledast$ (abobotulinumtoxinA) [prescribing information]. Boulogne-Billancourt: IpsenBiopharm Ltd; 2012. Accessed October 8. 2020.

14. XEOMIN (50/100/200) units powder for solution for injection summary of product characteristics. https://www medicines ie/ medicines/xeomin-50-100-200-units-powder-for-solution-for-injection-34858/spc. ; November 2019.

15. Spiegel LL, Ostrem JL, Bledsoe IO. FDA Approvals and Consensus Guidelines for Botulinum Toxins in the Treatment of Dystonia. Toxins (Basel). 2020; 12(5), doi: 10.3390/toxins12050332, indexed in Pubmed: 32429600.

16. Jost WH, Drużdż A, Pandey S, et al. Dose per muscle in cervical dystonia: pooled data from seven movement disorder centres. Neurol Neurochir Pol. 2021 [Epub ahead of print], doi: 10.5603/PJNNS. a2021.0005, indexed in Pubmed: 33471347.
17. Bentivoglio AR, Bagella CF, Albanese A. Botulinum toxin in movement disorders. In: Jankovic J, Tolosa E (eds) Parkinson's disease \& Movement Disorders 2015, 6th edn. Wolters Kluver, Philadelphia, pp. : 480-495.

18. Albanese A, Abbruzzese G, Dressler D, et al. Practical guidance for $\mathrm{CD}$ management involving treatment of botulinum toxin: a consensus statement. J Neurol. 2015; 262(10): 2201-2213, doi: 10.1007/ s00415-015-7703-x, indexed in Pubmed: 25877834.

19. Walter U, Mühlenhoff $\mathrm{C}$, Benecke R, et al. Frequency and risk factors of antibody-induced secondary failure of botulinum neurotoxin therapy. Neurology. 2020; 94(20): e2109-e2120, doi: 10.1212/ WNL.0000000000009444, indexed in Pubmed: 32332130.

20. Wu C, Xue F, Chang W, et al. Botulinum toxin type A with or without needle electromyographic guidance in patients with cervical dystonia. Springerplus. 2016; 5(1): 1292, doi: 10.1186/s40064-016-2967-x, indexed in Pubmed: 27547666.

21. Schramm A, Bäumer T, Fietzek U, et al. Relevance of sonography for botulinum toxin treatment of cervical dystonia: an expert statement. J Neural Transm (Vienna). 2015; 122(10): 1457-1463, doi: 10.1007/ s00702-014-1356-2, indexed in Pubmed: 25547861.

22. Tyślerowicz M, Jost WH. Injection into the longus colli muscle via the thyroid gland. Tremor Other Hyperkinet Mov (N Y). 2019; 9. indexed in Pubmed: 31867133. , doi: 10.7916/tohm.v0.718.

23. Kreisler A, Gerrebout C, Defebvre L, et al. Accuracy of non-guided versus ultrasound-guided injections in cervical muscles: a cadaver study. J Neurol. 2021 [Epub ahead of print], doi: 10.1007/s00415020-10365-w, indexed in Pubmed: 33399965.

24. Brumpt E, Aubry S, Vuillier F, et al. Anatomo-sonographic identification of the longissimus capitis and splenius cervicis muscles: principles for possible application to ultrasound-guided botulinum toxin injections in cervical dystonia. Surg Radiol Anat. 2021 [Epub ahead of print], doi: 10.1007/s00276-020-02646-w, indexed in Pubmed: 33459837.

25. Ko YD, Yun SIn, Ryoo D, et al. Accuracy of Ultrasound-Guided and Non-guided Botulinum Toxin Injection Into Neck Muscles Involved in Cervical Dystonia: A Cadaveric Study. Ann Rehabil Med. 2020; 44(5): 370-377, doi: 10.5535/arm.19211, indexed in Pubmed: 32986942.

26. Jost W. Torticaput versus Torticollis: Clinical Effects with Modified Classification and Muscle Selection. Tremor and Other Hyperkinetic Movements. 2019; 9(0), doi: 10.5334/tohm.474.

27. Hong JS, Sathe GG, Niyonkuru C, et al. Elimination of dysphagia using ultrasound guidance for botulinum toxin injections in cervical dystonia. Muscle Nerve. 2012; 46(4): 535-539, doi: 10.1002/mus.23409, indexed in Pubmed: 22987694.

28. Klingelhoefer L, Martino D, Martinez-Martin P, et al. Nonmotor symptoms and focal cervical dystonia: Observations from 102 patients. Basal Ganglia. 2014; 4(3-4): 117-120, doi: 10.1016/j. baga.2014.10.002.

29. Klingelhoefer L, Kaiser M, Sauerbier A, et al. Emotional well-being and pain could be a greater determinant of quality of life compared to motor severity in cervical dystonia. J Neural Transm (Vienna). 2021; 128(3): 305-314, doi: 10.1007/s00702-020-02274-z, indexed in Pubmed: 33146753.

30. Kuyper DJ, Parra V, Aerts S, et al. Nonmotor manifestations of dystonia: a systematic review. Mov Disord. 2011; 26(7): 1206-1217, doi: 10.1002/mds.23709, indexed in Pubmed: 21484874.

31. Slawek J, Friedman A, Potulska A, et al. Factors affecting the health-related quality of life of patients with cervical dystonia and the impact of botulinum toxin type A injections. Funct Neurol. 2007; 22(2): 95-100, indexed in Pubmed: 17637212. 
32. Berardelli I, Ferrazzano G, Pasquini M, et al. Clinical course of psychiatric disorders in patients with cervical dystonia. Psychiatry Res. 2015; 229(1-2): 583-585, doi: 10.1016/j.psychres.2015.07.076, indexed in Pubmed: 26239770.

33. Sitek EJ, Sołtan W, Wieczorek D, et al. Self-awareness of motor dysfunction in patients with Huntington's disease in comparison to Parkinson's disease and cervical dystonia. J Int Neuropsychol Soc. 2011; 17(5): 788-795, doi: 10.1017/S1355617711000725, indexed in Pubmed: 21729402.

34. Stamelou M, Edwards M, Hallett M, et al. Stamelou M, Edwards M, Hallett M, Bathia $\mathrm{K}$, The non-motor syndrome of primary dystonia: clinical and pathophysiological implications. Brain. 2012; 135(6): 1668-81.

35. Jost WH, Hefter H, Stenner A, et al. Rating scales for cervical dystonia: a critical evaluation of tools for outcome assessment of botulinum toxin therapy. J Neural Transm (Vienna). 2013; 120(3): 487-496, doi: 10.1007/s00702-012-0887-7, indexed in Pubmed: 22899277.

36. Supnet ML, Acuna P, Carr SJ, et al. Isolated Cervical Dystonia: Management and Barriers to Care. Front Neurol. 2020; 11: 591418, doi: 10.3389/fneur.2020.591418, indexed in Pubmed: 33329340.

37. Berlot R, Bhatia KP, Kojović M. Pseudodystonia: A new perspective on an old phenomenon. Parkinsonism Relat Disord. 2019; 62: 44-50, doi: 10.1016/j.parkreldis.2019.02.008, indexed in Pubmed: 30819557.

38. Dulski J, Sławek J. Fibrodysplasia ossificans progressiva as a form of pseudodystonia. Parkinsonism Relat Disord. 2020; 77: 180-181, doi: 10.1016/j.parkreldis.2020.01.013, indexed in Pubmed: 32046912.

39. Hefter H, Schomaecker I, Schomaecker M, et al. Disease Progression of Idiopathic Cervical Dystonia in Spite of Improvement After Botulinum Toxin Therapy. Front Neurol. 2020; 11: 588395, doi: 10.3389/ fneur.2020.588395, indexed in Pubmed: 33281726.

40. Marciniec M, Szczepańska-Szerej A, Rejdak K. Cervical dystonia: factors deteriorating patient satisfaction of long-term treatment with botulinum toxin. Neurol Res. 2020; 42(11): 987-991, doi: 10.1080/01616412.2020.1796430, indexed in Pubmed: 32693754.

41. Marciniec M, Szczepańska-Szerej A, Popek-Marciniec S, et al. Pain incidence in cervical dystonia is determined by the disease phenotype. J Clin Neurosci. 2020; 79: 133-136, doi: 10.1016/j.jocn.2020.07.069, indexed in Pubmed: 33070882.
42. Comella C, Ferreira JJ, Pain E, et al. Patient perspectives on the therapeutic profile of botulinum neurotoxin type A in cervical dystonia. J Neurol. 2021; 268(3): 903-912, doi: 10.1007/s00415-020-10217-7, indexed in Pubmed: 32939574.

43. Dressler D. Five-year experience with incobotulinumtoxinA (Xeo$\min (囚)$ ): the first botulinum toxin drug free of complexing proteins. Eur J Neurol. 2012; 19(3): 385-389, doi: 10.1111/j.1468-1331.2011.03559.x, indexed in Pubmed: 22035051.

44. Esquenazi A, Delgado MR, Hauser RA, et al. Duration of Symptom Relief Between Injections for AbobotulinumtoxinA (Dysport $囚$ ) in Spastic Paresis and Cervical Dystonia: Comparison of Evidence From Clinical Studies. Front Neurol. 2020; 11: 576117, doi: 10.3389/ fneur.2020.576117, indexed in Pubmed: 33101184.

45. Marion MH, Humberstone M, Grunewald R, et al. British Neurotoxin Network recommendations for managing cervical dystonia in patients with a poor response to botulinum toxin. Pract Neurol. 2016; 16(4): 288-295, doi: 10.1136/practneurol-2015-001335, indexed in Pubmed: 26976927.

46. Moro E, LeReun C, Krauss JK, et al. Efficacy of pallidal stimulation in isolated dystonia: a systematic review and meta-analysis. Eur J Neurol. 2017; 24(4): 552-560, doi: 10.1111/ene.13255, indexed in Pubmed: 28186378.

47. Ravindran K, Ganesh Kumar N, Englot DJ, et al. Deep Brain Stimulation Versus Peripheral Denervation for Cervical Dystonia: A Systematic Review and Meta-Analysis. World Neurosurg. 2019; 122: e940-e946, doi: 10.1016/j.wneu.2018.10.178, indexed in Pubmed: 30419402.

48. Kaelin-Lang A, You H, Burgunder JM, et al. Bilateral pallidal stimulation improves cervical dystonia for more than a decade. Parkinsonism Relat Disord. 2020; 81: 78-81, doi: 10.1016/j.parkreldis.2020.10.028, indexed in Pubmed: 33075700.

49. Wang J, Li J, Han L, et al. Selective peripheral denervation for the treatment of spasmodic torticollis: long-term follow-up results from 648 patients. Acta Neurochir (Wien). 2015; 157(3): 427-33; discussion 433, doi: 10.1007/s00701-015-2348-1, indexed in Pubmed: 25616622.

50. Cohen-Gadol AA, Ahlskog JE, Matsumoto JY, et al. Selective peripheral denervation for the treatment of intractable spasmodic torticollis: experience with 168 patients at the Mayo Clinic. J Neurosurg. 2003; 98(6): 1247-1254, doi: 10.3171/jns.2003.98.6.1247, indexed in Pubmed: 12816272. 